\title{
SUFFICIENT CONDITION FOR LOCAL INVERTIBILITY OF SPATIO-TEMPORAL 4D B-SPLINE DEFORMATIONS
}

\author{
Se Young Chun ${ }^{1}$, Colas Schretter ${ }^{2}$, and Jeffrey A. Fessler ${ }^{3}$ \\ ${ }^{1}$ Massachusetts General Hospital and Harvard Medical School, USA \\ ${ }^{2}$ RWTH Aachen University, Aachen, Germany \\ ${ }^{3}$ University of Michigan, Ann Arbor, USA
}

\begin{abstract}
Recent advances in medical imaging technologies have made 4D image sequences available in clinical routine. As a consequence, image registration techniques are evolving from alignment of pairs of static volumetric images to spatiotemporal registration of dynamic (4D) images. Since the elastic image registration problem is ill-posed, additional prior information or constraints are usually required to regularize the problem. This work proposes to enforce local invertibility (diffeomorphism) of 4D deformations. A novel sufficient condition for local invertibility over continuous space and time is proposed and a practical regularization prior is designed from the theory. The method has been applied to an image registration (motion tracking) of a dynamic 4D CT image sequence. Results show that using proposed regularizer leads to deformations that are more plausible for respiratory motion than the standard approach without additional temporal regularization.
\end{abstract}

Index Terms - 4D deformation, local invertibility, diffeomorphism, sufficient condition, image registration

\section{INTRODUCTION}

Nonrigid image registration has been investigated frequently for many medical imaging applications because patient motion may not be able to be described rigidly. Since usual nonrigid image registration problems are ill-posed, there has been much effort to regularize this inverse problem. There are many regularization criteria that have been researched [1].

Diffeomorphic or invertible deformation is one of the most popular regularization rules among nonrigid image registration. Since most motions for medical imaging applications are reversible, it is natural to require deformations to be diffeomorphic or invertible. There are three different ways to exploit this criteria: using necessary condition $[2,3]$, sufficient condition [4, 5, 6], and limited exact condition [7]. These are methods aligning pairs of static volumetric images.

This work is supported in part by 1P01 CA87634.
Nonrigid image registration has been used for registering a sequence of images and diffeomorphism has been desired to be used in many different medical imaging applications [8, 9, 10]. De Craene et al. [11] used the work of Beg et al. [12] to enforce diffeomorphism on spatio-temporal 4D deformations based on a chain of small and smooth deformations. In this approach, there is no exact constraint for small and smooth deformations. Rueckert et al. [13] used a chain of transformations that satisfy Choi's sufficient condition [4] for invertible B-spline deformations. We proposed to use larger constraints [6] to reduce the number of transformations. However, these sufficient conditions are valid only for $2 \mathrm{D}$ or $3 \mathrm{D}$ B-spline deformations.

This paper addresses the local invertibility of spatiotemporal 4D B-spline deformations. We provide a simple example to show that enforcing the local invertibility at each time sample does not enforce the local invertibility over the whole continuous time. Then, we propose a sufficient condition that guarantees the local invertibility of spatio-temporal 4D B-spline deformations. We show the image registration (motion tracking) result of a 4D image sequence onto a 3D single volume image using 4D CT images of a real patient based on this sufficient condition.

\section{BACKGROUND}

\subsection{Mathematical model for nonrigid transformation}

A 4D nonrigid transformation $\underline{T}: \mathbf{R}^{3} \times \mathbf{R} \rightarrow \mathbf{R}^{3} \times \mathbf{R}$ can be written

$$
\underline{T}(\underline{r}, t)=[\underline{r}+\underline{d}(\underline{r}, t)]
$$

where $\underline{r}=(x, y, z)^{T}$ and $\underline{d}(\underline{r}, t)$ is a deformation or a displacement. An identity transformation $t$ in (1) is added to define a composition of transformations. If one put a constant instead of $t$ at the last row of $\underline{T}$, then one can map a 3D image sequence onto a single 3D image. We model the 3D deformation $\underline{d}=\left(d^{x}, d^{y}, d^{z}\right)^{T}$ with the time axis using a tensor 
product of $n$ th-order B-splines as follows:

$$
\begin{aligned}
d^{q}(\underline{r}, t)= & \sum_{i j k l} c_{i j k l}^{q} \beta\left(x / m_{x}-i\right) \beta\left(y / m_{y}-j\right) \\
& \cdot \beta\left(z / m_{z}-k\right) \phi\left(t / m_{t}-l\right),
\end{aligned}
$$

where $q \in\{x, y, z\}, m_{q}$ is knot spacing in $q$ direction, $\beta$ is a $n$ th-order B-spline basis, and $\phi$ is a basis that satisfies the following conditions:

$$
\begin{array}{cl}
\sum_{l} \phi\left(t / m_{t}-l\right)=1, & \forall t \\
\phi\left(t / m_{t}-l\right) \geq 0, & \forall t .
\end{array}
$$

The goal in 4D image registration is to estimate the deformation coefficients $\underline{c}=\left\{c_{i, j, k, l}^{q}\right\}$ by maximizing a similarity metric $\Psi$ :

$$
\underline{\hat{c}}=\arg \max _{\underline{c}} \Psi[g(\cdot), f(\underline{T}(\cdot ; \underline{c}))]
$$

where $g(\underline{r}, t)$ denote a 4D image sequence and $f(\underline{r})$ denote a $3 \mathrm{D}$ or $4 \mathrm{D}$ image.

To help stabilize the estimation, and to have physically plausible deformations, often we would like to ensure that the estimated coefficients $\underline{\hat{c}}$ correspond to a diffeomorphic transformation $\underline{T}$. The methods in this paper are applicable to any similarity metric; for a survey of such metrics, see [14].

\subsection{Local invertibility of deformations over time}

If we enforce the determinant values of the $3 \times 3$ Jacobian matrix $\nabla \underline{T}(\underline{r}, t)$ corresponding to $\underline{r}$ to be positive for all $\underline{r}$ and $t$, we can truly achieve local invertibility of deformations in the continuous 4D space. However, enforcing it at discrete time points does not guarantee that the determinant values inbetween these discrete time points are positive.

For a linear transformation $\underline{T}(\underline{r}, t)$, assume that we have two invertible transformations at $t=1$ and $t=2$ such that

$$
\underline{T}(x, y, 1)=\left[\begin{array}{ll}
5 & 6 \\
4 & 5
\end{array}\right]\left[\begin{array}{l}
x \\
y
\end{array}\right]
$$

and

$$
\underline{T}(x, y, 2)=\left[\begin{array}{ll}
5 & 4 \\
6 & 5
\end{array}\right]\left[\begin{array}{l}
x \\
y
\end{array}\right] .
$$

We can easily show that $|\nabla \underline{T}(x, y, 1)|=|\nabla \underline{T}(x, y, 2)|=$ $1>0$. However, if we interpolate a $\underline{T}(x, y, t)$ value at $t=1 / 2$ by using a usual linear interpolation, we can show that $\underline{T}(x, y, 1 / 2)=\underline{T}(x, y, 1) / 2+\underline{T}(x, y, 2) / 2$ and $|\nabla \underline{T}(x, y, 1 / 2)|=0$ for all $(x, y)$. Therefore, $\left|\nabla \underline{T}\left(\underline{r}, t_{n}\right)\right|>$ 0 for all $\underline{r}$ and $t_{1}, \cdots, t_{N}$ does not imply that $|\nabla \underline{T}(\underline{r}, t)|>0$ for all $\underline{r}$ in general when one has invertible transformations at discrete time points and one uses an overlapped time bases for the time axis interpolation.

\section{LOCAL INVERTIBILITY CONDITION FOR SPATIO-TEMPORAL 4D DEFORMATIONS}

This section extends the lemmas and theorems in [6] for 4D deformations in the continuous space and time domain.

Lemma 1. If $b_{m} \leq c_{i+1, j, k, l}^{q}-c_{i, j, k, l}^{q} \leq b_{M}$ for $\forall i, j, k, l$, then $b_{m} / m_{x} \leq \partial d^{q}(\underline{r}, t) / \partial x \leq b_{M} / m_{x}$ for $\forall \underline{r}$ where $q \in$ $\{x, y, z\}$ Similarly, if $b_{m} \leq c_{i, j+1, k, l}^{q}-c_{i, j, k, l}^{q} \leq b_{M}$ for $\forall i, j, k, l$, then $b_{m} / m_{y} \leq \partial d^{q}(\underline{r}, t) / \partial y \leq b_{M} / m_{y}$ and if $b_{m} \leq c_{i, j, k+1, l}^{q}-c_{i, j, k, l}^{q} \leq b_{M}$ for $\forall i, j, k, l$, then $b_{m} / m_{z} \leq$ $\partial d^{q}(\underline{r}, t) / \partial z \leq b_{M} / m_{z}$ for $\forall \underline{r}$ respectively.

Proof. For $h(x)=\sum_{i} c_{i} \beta^{n}\left(x / m_{x}-i\right)$, by using $\partial \beta^{n}(x) / \partial x=$ $\beta^{n-1}(x+1 / 2)-\beta^{n-1}(x-1 / 2)[15]$

$$
\frac{\partial}{\partial x} h(x)=\sum_{i}\left(c_{i}-c_{i-1}\right) \beta^{n-1}\left(x / m_{x}-i+1 / 2\right) / m_{x} .
$$

Similarly as in [6], using the constraints $b_{m} \leq c_{i+1, j, k, l}^{q}-$ $c_{i, j, k, l}^{q} \leq b_{M}$ and the nonnegativeness of $\beta^{n}(\cdot)$ and $\phi(\cdot)$, we have the bounds

$$
\begin{aligned}
& \frac{\partial}{\partial x} d^{q}(\underline{r}, t) \leq b_{M} / m_{x} \sum_{i} \beta^{n-1}\left(x / m_{x}-i+1 / 2\right) \cdot \sum_{j} \beta^{n}\left(y / m_{y}-j\right) \sum_{k} \beta^{n}\left(z / m_{z}-k\right) \\
& \cdot \sum_{l} \phi\left(t / m_{t}-l\right) \\
& \leq b_{M} / m_{x}
\end{aligned}
$$

where $\sum_{i} \beta^{n}\left(x / m_{x}-i\right)=1$ and $\sum_{l} \phi\left(t / m_{t}-l\right)=1$. Similarly, $\partial d^{q}(\underline{r}, t) / \partial x \geq b_{m} / m_{x}$. The inequalites of $\partial d^{q}(\underline{r}, t) / \partial y$ and $\partial d^{q}(\underline{r}, t) / \partial z$ can also be proved in similar ways.

Therefore, the sufficient condition for the local invertibility of deformations [6] also holds for 4D deformation models (2) with a basis $\phi(\cdot)$ for continuous time:

Theorem 1. Suppose $0 \leq k_{q}<\frac{1}{2}$ for $q \in\{x, y, z\}$. Define:

$$
\begin{aligned}
C_{5} \triangleq\{\underline{c}: & -m_{x} k_{x} \leq c_{i+1, j, k, l}^{x}-c_{i, j, k, l}^{x} \leq m_{x} K_{x}, \\
& -m_{y} k_{y} \leq c_{i, j+1, k, l}^{y}-c_{i, j, k, l}^{y} \leq m_{y} K_{y}, \\
& -m_{z} k_{z} \leq c_{i, j, k+1, l}^{z}-c_{i, j, k, l}^{z} \leq m_{z} K_{z}, \\
& \left|c_{i+1, j, k, l}^{q}-c_{i, j, k, l}^{q}\right| \leq m_{q} k_{q} \text { for } q=y, z, \\
& \left|c_{i, j+1, k, l}^{q}-c_{i, j, k, l}^{q}\right| \leq m_{q} k_{q} \text { for } q=x, z, \\
& \left.\left|c_{i, j, k+1, l}^{q}-c_{i, j, k, l}^{q}\right| \leq m_{q} k_{q} \text { for } q=x, y, \forall i, j, k, l\right\} .
\end{aligned}
$$

In (1), if $\underline{c} \in C_{5}$ then $1-\left(k_{x}+k_{y}+k_{z}\right) \leq|\nabla \underline{T}(\underline{r}, t)| \leq$ $\left(1+K_{x}\right)\left(1+K_{y}\right)\left(1+K_{z}\right)+\left(1+K_{x}\right) k_{y} k_{z}+k_{x}\left(1+K_{y}\right) k_{z}+$ $k_{x} k_{y}\left(1+K_{z}\right) \forall \underline{r} \in \mathbf{R}^{3}$ and $\forall t \in \mathbf{R}$. Moreover, if $k_{x}+$ $k_{y}+k_{z}<1$, then the transformation (1) is locally invertible everywhere. 
Note that this sufficient condition for 4D deformations is the same as the condition for 3D deformations in [6] and it does not require temporal regularization. It is because the sufficient condition in [6] is a convex set. As long as a temporal basis $\phi(\cdot)$ satisfies (3) and (4) (e.g. $n$th order B-spline basis), we can use the same sufficient condition for local invertibility of both $3 \mathrm{D}$ and $4 \mathrm{D}$ deformations.

\section{OPTIMIZATION BASED ON PROPOSED SUFFICIENT CONDITION}

One may use constrained optimization with the proposed sufficient condition (hard constraints) or use a simple penalty based on this condition (soft constraints) [6].

\subsection{Lagrange multiplier}

One can use a Lagrange multiplier method to perform image registration (5) with the proposed sufficient condition for the local invertibility of 4D deformations. Sdika described the constrained optimization with a Lagrange multiplier for image registration [3]. This method with the proposed sufficient condition enforces the local invertibility of 4D deformations and the estimated B-spline coefficients $\underline{\hat{c}} \in C_{5}$.

Since it is a sufficient condition, the constraint may not contain the true deformations. A concatenation of several transformations that belong to this constraint may be able to describe more complicated deformations $[13,6]$, i.e.,

$$
\underline{T}_{n} \circ \underline{T}_{n-1} \circ \cdots \circ \underline{T}_{1}
$$

Since the proposed sufficient condition guarantees the local invertibility of deformations over continuous space and time, the composition of them also guarantees the local invertibility.

\subsection{Simple regularizer}

Another way to use this proposed sufficient condition is to relax the invertibility condition by using a penalty method [2, 6]. In a penalty method we maximize an objective function that is the similarity metric minus a penalty function that encourages the invertibility condition, but does not enforce it strictly.

Being based on the somewhat restrictive solution space $C_{5}$, the penalty method can "encourage" the local invertibility on the whole continuous time and space with a fast and memory efficient implementation [6]. This implementation is usually possible because $C_{5}$ does not require additional Bspline interpolations beyond the interpolations needed for the data fitting term.

\section{SIMULATION RESULTS}

We aligned a sequence of 4D CT images $(128 \times 128 \times 64 \times$ 10, Fig. 1) to a single $3 \mathrm{D}$ reference image with a constrained

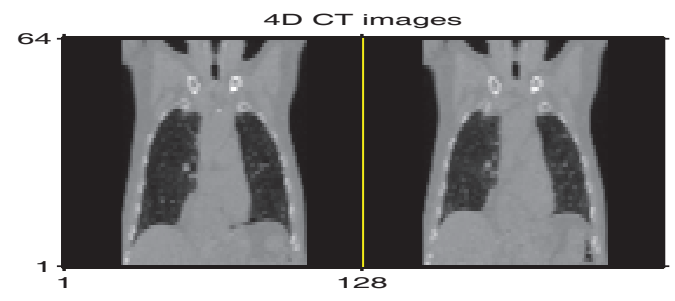

Fig. 1. 4D CT image sequence $(128 \times 128 \times 64 \times 10)$.

optimization. For 4D deformations, we chose $m_{x}=m_{y}=$ $m_{z}=4$ and $m_{t}=2$. We also performed pairwise 3D image registrations from every other motion phases to a reference frame. B-spline interpolation consumes most of computation time [6]. An efficient interpolation [15] and a parallelization with GPU can speed up the computation significantly.

Fig. 2 shows the difference images between a target image and source, deformed images by $3 \mathrm{D}$ and $4 \mathrm{D}$. It seems that deformations in 3D and 4D sufficient conditions can align sequence of 4D CT images in Fig. 1. Since 3D sufficient condition contains our proposed 4D sufficient condition by allowing any interpolation instead of allowing only B-spline interpolation, the norm of image difference between deformed and target images for 3D is smaller than for 4D.
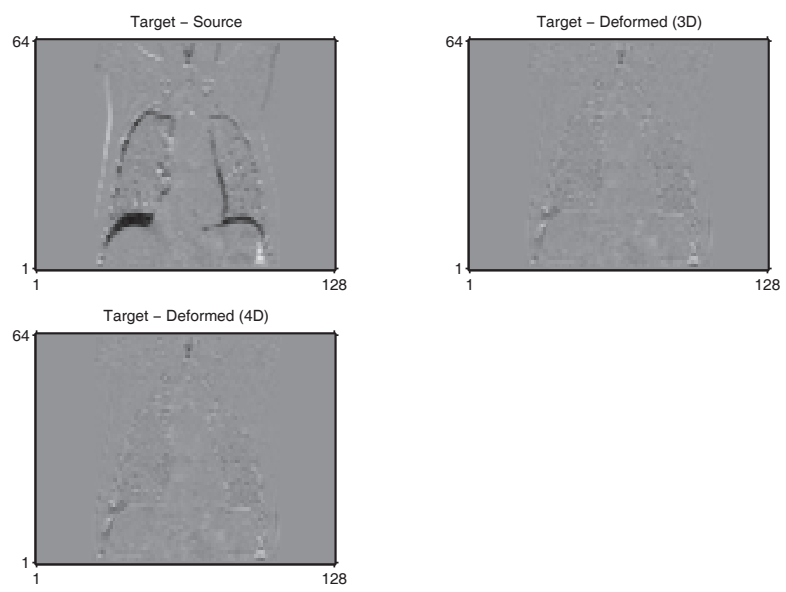

Fig. 2. Difference images between a target (frame 7) and a source (left, up), a deformed image by 3D deformation (right, up), a deformed image by 4D deformation (left, down).

Fig. 3 shows the z-direction deformations of 3D and 4D over a breathing cycle at 5 different voxels (anatomical locations). This 4D result shows more plausible for a breathing motion than $3 \mathrm{D}$ result without additional temporal regularization.

We interpolated deformations by using cubic B-spline for 3D and 4D deformations (7 points between two time frames). Fig. 4 shows Jacobian determinant values at control points (square and circle) and inbetween values at 2 different voxels 

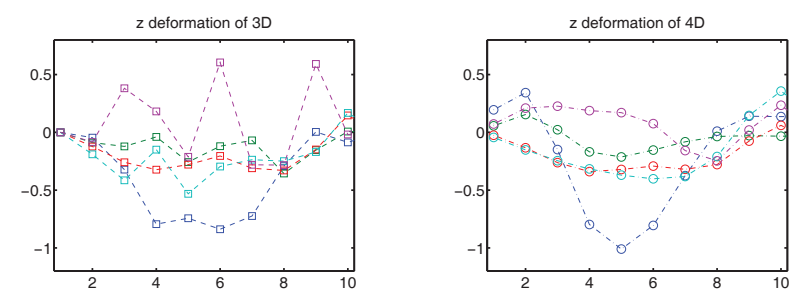

Fig. 3. Z direction of 3D (left) and 4D (right) deformations over one cycle of breathing at 5 different voxels.

(anatomical locations). 3D result shows that the Jacobian determinant of inbetween values may go much lower than values at control points. The minimum Jacobian determinant values of $3 \mathrm{D}$ and $4 \mathrm{D}$ deformations at control frames were 0.30 and 0.31 . However, the minimum Jacobian determinant values of 3D and 4D deformations over all interpolated time frames were 0.25 and 0.30 . Smoothness of interpolator for $3 \mathrm{D}$ deformations prevented the determinant values decrease significantly, but without any theoretical justification to control inbetween values.
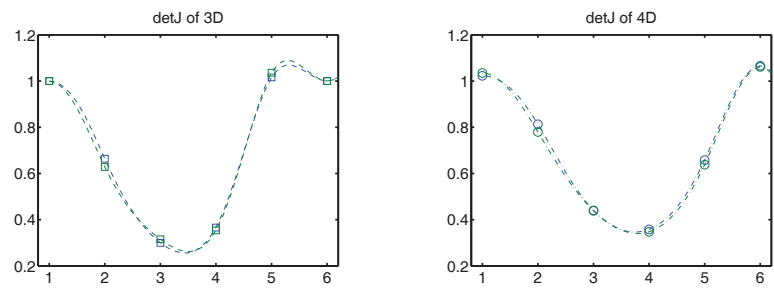

Fig. 4. Jacobian determinants at control points (square, circle) of 3D (left) and 4D (right) over time and inbetween values at 2 different voxels.

\section{DISCUSSION}

Enforcing the local invertibility of 4D deformations at discrete times does not guarantee the local invertibility of 4D deformations over continuous time. One may achieve local invertibility using additional temporal regularization or smoothing, but it is challenging to determine how much one has to regularize. Instead of using sufficiently small 4D deformations for local invertibility, our proposed sufficient condition provides a guideline to achieve local invertibility of spatio-temporal 4D B-spline deformations over continuous space and time. More experimental results such as comparing with ground truth motions is necessary.

\section{REFERENCES}

[1] W R Crum, T Hartkrns, and D L G Hill, "Non-rigid image registration: theory and practice," The British Journal of Radiology, vol. 77, pp. S140-S153, 2004.
[2] J. Kybic, P. Thevenaz, A. Nirkko, and M. Unser, "Unwarping of unidirectionally distorted EPI images," IEEE Trans. Med. Imag., vol. 19, no. 2, pp. 80-93, Feb. 2000.

[3] M. Sdika, "A fast nonrigid image registration with constraints on the jacobian using large scale constrained optimization," IEEE Trans. Med. Imag., vol. 27, no. 2, pp. 271-81, Feb. 2008.

[4] Y. Choi and S. Lee, "Injectivity conditions of 2D and 3D uniform cubic B-spline functions," Graphical Models, vol. 62, no. 6, pp. 411-27, 2000.

[5] G. K. Rohde, A. Aldroubi, and B. M. Dawant, "The adaptive bases algorithm for intensity-based nonrigid image registration," IEEE Trans. Med. Imag., vol. 22, no. 11, pp. 1470-9, Nov. 2003.

[6] S. Y. Chun and J. A. Fessler, "A simple regularizer for B-spline nonrigid image registration that encourages local invertibility," IEEE J. Sel. Top. Sig. Proc., vol. 3, no. 1, pp. 159-69, Feb. 2009, Special Issue on Digital Image Processing Techniques for Oncology.

[7] V. Noblet, C. Heinrich, F. Heitz, and J-P. Armspach, “3-D deformable image registration: a topology preservation scheme based on hierarchical deformation models and interval analysis optimization," IEEE Trans. Im. Proc., vol. 14, no. 5, pp. 55366, May 2005.

[8] K. Taguchi, Z. Sun, W. P. Segars, E. K. Fishman, and B. M. W. Tsui, "Image-domain motion compensated time resolved 4D cardiac CT," in Proc. SPIE 6510, Medical Imaging 2007: Phys. Med. Im., 2007, p. 651016.

[9] Colas Schretter, Christoph Neukirchen, Matthias Bertram, and Georg Rose, "Correction of some time-dependent deformations in parallel-beam computed tomography," in 5th IEEE International Symposium on Biomedical Imaging (ISBI), 2008, pp. $764-767$.

[10] E. Hansis, H. Schomberg, K. Erhard, O. Dössel, and M. Grass, "Four-dimensional cardiac reconstruction from rotational x-ray sequences: first results for 4D coronary angiography," in Proc. SPIE 7258, Medical Imaging 2009: Phys. Med. Im., 2009, p. $72580 \mathrm{~B}$.

[11] Mathieu De Craene, Oscar Camara, Bart H. Bijnens, and Alejandro F. Frangi, "Large diffeomorphic FFD registration for motion and strain quantification from 3D-US sequences," in Functional Imaging and Modeling of the Heart, 2009, vol. 5528, pp. 437-446.

[12] M. F. Beg, M. I. Miller, A. Trouve, and L. Younes, "Computing large deformation metric mappings via geodesic flows of diffeomorphisms," Intl. J. Comp. Vision, vol. 61, no. 2, pp. 139-57, Feb. 2005.

[13] D. Rueckert, P. Aljabar, R. A. Heckemann, J. V. Hajnal, and A. Hammers, "Diffeomorphic registration using B-splines," in Medical Image Computing and Computer-Assisted Intervention, 2006, pp. 702-9.

[14] D. L. G. Hill, P. G. Batchelor, M. Holden, and D. J. Hawkes, "Medical image registration," Phys. Med. Biol., vol. 46, no. 3, pp. R1-47, Mar. 2001.

[15] M. Unser, A. Aldroubi, and M. Eden, "B-spline signal processing: Part I-theory," IEEE Trans. Sig. Proc., vol. 41, no. 2, pp. 821-33, Feb. 1993. 